\title{
New connections between 4D and 5D black holes
}

\section{Citation}

Gaiotto, Davide, Andrew Strominger, and Xi Yin. 2006. "New Connections between 4D and 5D Black Holes." Journal of High Energy Physics 2006 (2): 024-024. https:// doi.org/10.1088/1126-6708/2006/02/024.

\section{Permanent link}

http://nrs.harvard.edu/urn-3:HUL.InstRepos:41417359

\section{Terms of Use}

This article was downloaded from Harvard University's DASH repository, and is made available under the terms and conditions applicable to Other Posted Material, as set forth at http:// nrs.harvard.edu/urn-3:HUL.InstRepos:dash.current.terms-of-use\#LAA

\section{Share Your Story}

The Harvard community has made this article openly available.

Please share how this access benefits you. Submit a story.

\section{Accessibility}


hep-th/0503217

\title{
New Connections Between 4D and 5D Black Holes
}

\author{
Davide Gaiotto* , Andrew Strominger* and Xi Yin* \\ Center of Mathematical Sciences \\ Zhejiang University, Hangzhou 310027 China
}

\begin{abstract}
A simple equality is proposed between the BPS partition function of a general 4D IIA Calabi-Yau black hole and that of a 5D spinning M-theory Calabi-Yau black hole. Combining with recent results then leads to a new relation between the 5D spinning BPS black hole partition function and the square of the $N=2$ topological string partition function.
\end{abstract}

* Permanent address: Jefferson Physical Laboratory, Harvard University, Cambridge, MA, USA. 


\section{Contents}

1. Introduction . . . . . . . . . . . . . . . . . . . . . . . . . . . . 1

2. $\mathrm{M} \rightarrow \mathrm{IIA}$. . . . . . . . . . . . . . . . . . . . . . . . . . . 2

3. D6-D2-D0 Entropy . . . . . . . . . . . . . . . . . . . . . . . . . 4

4. Spinning black hole and topological strings . . . . . . . . . . . . . . . . . . . . 5

5. The D6-D4-D2-D0 system . . . . . . . . . . . . . . . . . . . . . . . . 6

5.1. $p^{0}=1 \quad$. . . . . . . . . . . . . . . . . . . . . . . . . . . . . . . . . 7

5.2. $p^{0}>1 \quad$. . . . . . . . . . . . . . . . . . . . . . . . . . . . . . . . . . . . . . . 9

Appendix A. Supergravity solutions of spinning black hole in Taub-NUT space . . . . . . 9

\section{Introduction}

Investigations of BPS black hole in string theory has shown them to be a gold mine for deep and surprising physical and mathematical insights. In this paper we continue these investigations in proposing and giving evidence for a simple and direct connection between a certain BPS partition function $Z_{5 D}$ of the general $5 \mathrm{D}$ spinning BPS black hole in a Calabi-Yau compactification of M-theory and $Z_{4 D}$ of the general 4D BPS black hole in a Calabi-Yau compactification of the IIA theory. Invoking prior results [1] then leads to a simple non-linear relation between $Z_{5 D}$ and topological string partition function $Z_{t o p}$.

We begin in section 2 by deriving the basic 4D-5D connection. Exact 5D supersymmetric solutions were found in [2] which can be described as a $5 \mathrm{D}$ black hole with $S U(2)_{L}$ spin $J_{L}^{3}$ and M2 charges $q_{A}^{5 D}$ sitting at the center of a charge $p^{0}$ Taub-NUT. Since TaubNUT is locally asymptotic to flat $R^{3} \times S^{1}$ this implements a $5 \rightarrow 4$ compactification. When the compactification radius $R$, a modulus of the Taub-NUT solution, becomes small the $4 \mathrm{D}$ picture becomes appropriate. We show that in the $4 \mathrm{D}$ picture we have a black hole with D6-D2-D0 charges $\left(p^{0}, \frac{q_{A}^{5 D}}{p^{0}}, \frac{2 J_{L}^{3}}{\left(p^{0}\right)^{2}}\right)$, and vanishing D4 charge $p^{A}=0$.

In section 3 we argue that an appropriate BPS partition function (i.e. index) $Z$ should not depend on the radius $R$, yielding an equality of the form $Z_{4 D}=Z_{5 D}$ with a certain relation between the arguments. The microscopic description for many (but not all) 5D spinning black holes is known [3,4]. Hence this 5D-4D relation gives a microscopic description of $4 \mathrm{D}$ black holes for many cases in which it had previously been unknown. As a check these relations are found to correctly, and in a rather intricate manner, reproduce the entropy formula at leading order. 
In section 4 we use this and a prior result [1] to give a relation of the form 1

$$
Z_{5 D}(\mu)=\left|Z_{\text {top }}\left(g_{\text {top }}=\frac{8 \pi^{2}}{\mu}\right)\right|^{2}
$$

between the BPS 5D black hole partition function and the topological string partition function. Here $\mu$ is the potential for $S U(2)_{L}$ spin and $g_{t o p}$ is the topological string coupling constant. (1.1] is quite different from the linear relation of [7,8] between a certain 5D BPS partition function and $Z_{t o p}$ and involving $g_{t o p} \sim \mu$. Combining (1.1) with the results of [7,8] potentially leads to a non-trivial relation between $Z_{t o p}$ at different points in the moduli space.

In section 5 the result is generalized to include general D4 charge $p^{A}$. From the 5D M-theory perspective this involves turning on a four form $F^{(4)} \sim \omega_{N U T} \wedge p^{A} \alpha_{A}$, where $\omega_{N U T}$ is a harmonic Taub-NUT two form and $\alpha_{A}$ is an integral basis of harmonic CalabiYau two forms. The 4D partition function for any set of D-brane charges may then be identified with that of a spinning 5D black hole in this Taub-NUT-flux background. This identification is again shown to intricately yield the correct leading-order entropy.

\section{2. $\mathrm{M} \rightarrow$ IIA}

Consider $p^{0}$ D6 branes wrapping a Calabi-Yau space $X$ in a IIA string compactification. In the M-theory picture this is described as the product of a Taub-NUT space with $X:$

$$
d s_{M}^{2}=\left(1+\frac{p^{0} R}{r}\right) d \vec{r}^{2}+R^{2}\left(1+\frac{p^{0} R}{r}\right)^{-1}\left(d x^{11}+p_{0} \cos \theta d \phi\right)^{2}+d s_{X}^{2}-d t^{2}
$$

where $x^{11} \sim x^{11}+4 \pi$. The Taub-NUT geometry has a $U(1)_{L} \times S U(2)_{R}$ isometry, where the $U(1)_{L}$ generates $x^{11}$ translations. The radius $R$ here is related to the ten-dimensional IIA coupling via

$$
R=g_{10}^{2 / 3}
$$

At strong coupling, or large $R$, there is a large region with $r \ll R$ in the core of the Taub-NUT geometry in which the 5D metric reduces to

$$
d s_{5}^{2}=\frac{p^{0} R}{r}\left(d r^{2}+r^{2} d \theta^{2}+r^{2} \sin ^{2} \theta d \phi^{2}+r^{2}\left(d x^{11} / p_{0}+\cos \theta d \phi\right)^{2}\right)-d t^{2}
$$

1 As discussed in [1] and [5] [6] there are a number of subtleties in interpreting the 4D version of this formula which of course also pertain here. 
This is the flat metric on $R^{4} / Z_{p^{0}}$ tensored with the time direction. For $p^{0}=1$ we simply have 5D Minkowski space.

Calabi-Yau compactifications of $\mathrm{M}$ theory to 5D admit a second set of supersymetric solutions with $U(1)_{L} \times S U(2)_{R}$ isometries. These are the $5 \mathrm{D}$ spinning black holes [4], characterized by membrane charges $q_{A}$ and angular momentum $J_{L}$ associated to the $U(1)_{L}$ isometry. Their characteristic size $r_{B H}$ grows as the square root of the graviphoton charge $\sqrt{Q}$ which in turn is proportional to the membrane charge $q_{A}$.

Let us now suppose that $\sqrt{Q} \ll R$ and $p^{0}=1$. Then we can make an approximate BPS solution by inserting the spinning black hole at the center of the $p^{0}=1$ Taub-NUT, symmetries aligned, well inside the region where the $R^{4}$ is flat. Aligning the symmetries requires the black hole to be exactly at the center of the Taub-NUT. In fact an exact solution of this form exists for all $Q, R$ [2] and is reproduced in the appendix. Of course for large $\sqrt{Q} \gg R$ it can no longer be described as a black hole in the center of TaubNUT, but this is irrelevant for our purposes since the BPS quantities we consider should be independent of $R$.

At distances large compared to $R$, this solution is effectively a spherically symmetric black hole in a four dimensional IIA compactification carrying D6 charge $p^{0}=1$, and D2 charge $q_{A}$. In addition $J_{L}$, which is the eigenvalue of $U(1)_{L}$ rotations, becomes proportional to D0 charge $q_{0}$, since $U(1)_{L}$ generates $x^{11}$ translations. To get the proportionality factor, consider an orbit of the asymptotic $U(1)_{L}$ in the $S^{3}$ near the tip $\mathbf{R}^{4}$. The angular momentum in the 1-2 plane $J_{1}$ and that in the 3-4 plane $J_{2}$ are related to $J_{L}, J_{R}$ by $J_{1}=J_{L}+J_{R}, J_{2}=J_{L}-J_{R}$. An orbit of the $U(1)_{L}$ is a helix going along a circle in the 1-2 plane and a circle in the 3-4 plane at the same time. The wave function of angular momentum $\left(J_{L}, J_{R}=0\right)$ picks up a factor $e^{2 \pi i\left(J_{1}+J_{2}\right)}=e^{4 \pi i J_{L}}$ as one goes around the $S^{1}$ orbit. Therefore we conclude

$$
q_{0}=2 J_{L}
$$

A similar construction works for integral $p^{0}>1$. We simply take the exact $5 \mathrm{D}$ solution and quotient it by the $Z_{p^{0}}$ subgroup of the $U(1)_{L}$ isometry, which acts freely outside the horizon. At infinity, this quotients the Kaluza-Klein circle and changes its radius from $R$ to $\frac{R}{p^{0}}$, while the topology of the $5 \mathrm{D}$ horizon becomes $S^{3} / Z_{p^{0}}$. The corresponding $4 \mathrm{D}$ black 
hole then has zero-brane charge 2

$$
q_{0}=\frac{2 J_{L}}{\left(p^{0}\right)^{2}} .
$$

Moreover, since the $S^{2} \times S^{1}$ at infinity over which the $4 \mathrm{D}$ charges are given as field strength integrals is divided by $p^{0}$, we have

$$
q_{A}=\frac{q_{A}^{5 D}}{p^{0}}
$$

\section{D6-D2-Do Entropy}

The preceding classical construction suggests the quantum conjecture that the supersymmetric partition function of a $4 \mathrm{D}$ black hole with D-brane charges $\left(p^{0}, 0, q_{A}, q_{0}\right)$ is directly related to that of a $Z_{p^{0}}$ orbifold (which is trivial for $p^{0}=1$ ) of a $5 \mathrm{D}$ black hole with membrane charges $q_{A}$ and spin $q_{0} / 2$. A precise conjecture relating certain $4 \mathrm{D}$ and $5 \mathrm{D}$ supersymmetric indices will be made in the next section. In this section we will check the conjecture at the level of the leading semiclassical entropy.

The macroscopic entropy of a 5D spinning black hole is [9]

$$
S_{5 D B H}=2 \pi \sqrt{Q^{3}-J_{L}^{2}},
$$

where $Q^{3}=D_{A B C} Y^{A} Y^{B} Y^{C}$ with $Y^{A}$ 's satisfying $3 D_{A B C} Y^{B} Y^{C}=q_{A}^{5 D}$. A $4 \mathrm{D}$ black hole is obtained by inserting this $5 \mathrm{D}$ black hole in the center of Taub-NUT. For the special case $p^{0}=1$, we identify $J_{L}=q_{0} / 2$, and (3.1) becomes

$$
S_{4 D B H}\left(p^{0}=1\right)=2 \pi \sqrt{Q^{3}-\frac{1}{4}\left(q_{0}\right)^{2}} .
$$

This agrees precisely with the known $4 \mathrm{D}$ result for no D4 charges and $p^{0}=1$ [10].

This is to be expected: in the reduction from 5D supergravity to $4 \mathrm{D}$ supergravity the radius of the fifth dimension is identified with an appropriate combination of the $4 \mathrm{D}$ scalar moduli, and the Taub-NUT radius is the asymptotic value of that scalar modulus at infinity. The entropy of a 4D BPS black hole does not depend of the asymptotic values of the scalar moduli at infinity.

2 Writing the D0 charge schematically as a 4 D spatial integral $q_{0} \sim \int d^{4} \Sigma^{b} K^{a} T_{a b}$ of the $U(1)_{L}$ Killing field $K$ contracted with the stress tensor, one factor of $p^{0}$ comes from the division of the domain of the integrand, while the second comes from demanding that $K$ be normalized so as to generate unit translations of the Kaluza-Klein circle at infinity. 
Therefore, any microscopic accounting of a 5D black hole with charges $q_{A}$ directly descends to a microscopic accounting of a $4 \mathrm{D}$ black hole with D6 charge $p^{0}=1$, D4 charge $p^{A}=0$, and arbitrary D2-D0 charges $q_{A}, q_{0}$.

Now consider $p^{0}>1$. Dividing by $p^{0}$ divides the area and hence the entropy by $p^{0}$. Therefore, in terms of the parameters $J_{L}$ and $Q_{5 D}$ of the unquotiented 5D black hole the $4 \mathrm{D}$ entropy is

$$
S_{4 D B H}=\frac{2 \pi}{p^{0}} \sqrt{Q_{5 D}^{3}-J_{L}^{2}} .
$$

Using (2.5) and (2.6) then gives

$$
S_{4 D B H}=2 \pi \sqrt{p^{0} Q^{3}-\frac{1}{4}\left(p^{0} q_{0}\right)^{2}}
$$

in precise agreement with the $4 \mathrm{D}$ entropy formula for general nonzero D0, D2 and D6 charges [10].

For $p^{0}>1$ a microscopic accounting of 5D entropy does not descend so directly to an accounting of $4 \mathrm{D}$ entropy, because we still have to understand the effect of the $Z_{p^{0}}$ orbifold action on the dual quantum microsystem describing the black hole. The dual quantum microsystem is not known in general so we can't describe the orbifold action. In order to proceed we assume a microscopic picture of the kind discovered in [3, [4], in which the $U(1)_{L}$ corresponds to a conserved left-moving current of a $2 \mathrm{D} \mathrm{CFT}$. $Z_{p^{0}}$ is then an orbifold action, and the entropy is dominated by the "long string" of the maximally twisted sector. This

effectively increases the $2 \mathrm{D}$ central charge by a factor of $p^{0}$ so that $Q^{3} \rightarrow p^{0} Q^{3}$. At the same time the relation between worldvolume momentum and target space one is rescaled as well $q_{0} \rightarrow p^{0} q_{0}$, and we recover (3.4). With this assumption, any microscopic accounting of a $5 \mathrm{D}$ black hole with charges $q_{A}$ directly descends to a microscopic accounting of a 4D black hole with D4 charge $p^{A}=0$, and arbitrary D6-D2-D0 charges $p^{0}, q_{A}, q_{0}$. In section 5 we will relax the restriction $p^{A}=0$.

\section{Spinning black hole and topological strings}

We conjecture the exact relation between the partition function of $4 \mathrm{D}$ extremal black holes and 5D spinning black holes, as follows

$$
Z_{4 D}\left(\phi^{A}, \phi^{0}\right)=Z_{5 D}\left(\phi^{A}, 2 \phi^{0}+2 \pi i\right)
$$


where these partition functions are Witten indices of the form

$$
Z_{4 D}\left(\phi^{A}, \phi^{0}\right)=\operatorname{Tr}_{p^{0}=1, p^{A}=0}^{\prime}(-1)^{2 J^{3}} e^{-\phi^{A} q_{A}-\phi^{0} q_{0}-\beta H}
$$

and

$$
Z_{5 D}\left(\phi^{A}, \mu\right)=\operatorname{Tr}(-1)^{2 J_{L}^{3}+2 J_{R}^{3}} e^{-\phi^{A} q_{A}-\mu J_{L}^{3}-\beta H} .
$$

$\operatorname{Tr}^{\prime}$ here denotes the trace over all $4 \mathrm{D}$ states with the overall center-of-mass multiplet factored out 3 and $J^{3}$ generates a $4 \mathrm{D}$ spatial rotation. The $4 \mathrm{D}$ trace is restricted to the sector with $p^{0}=1$ and $p^{A}=0 . Z_{5 D}$ has IR divergences from black holes which fragment and separate: we regulate these by putting them in Taub-NUT space of radius $R$ which forces all black holes to sit at the center (where they do not break supersymmetry) 1 and then taking $R \rightarrow \infty$. Using $J^{3}(4 D)=J_{R}^{3}(5 D), 2 q_{0}=J_{L}^{3}(5 D)$ and the relation $Z_{4 D}=$ $\left|Z_{\text {top }}\right|^{2}$ of [1] we have for $Z_{5 D}$

$$
Z_{5 D}\left(\phi^{A}, \mu=2 \phi^{0}-2 \pi i\right)=Z_{4 D}\left(\phi^{A}, \phi^{0}\right)=\left|Z_{\text {top }}\left(g_{\text {top }}=\frac{8 \pi^{2}}{\mu}, t^{A}=\frac{2 \pi \phi^{A}}{\mu}\right)\right|^{2}
$$

Here $t^{A}$ are the Kähler moduli for the topological string, $\phi^{0}$ is understood to be real.

This relation can be generalized to $p^{0}>1$ and/or $p^{A} \neq 0$ (see the next section) but additional assumptions are required. (4.1) seems to be the simplest of the relations between 4D and 5D black holes.

\section{The D6-D4-D2-D0 system}

In this section we generalize our construction to 4D extremal black hole of generic charges $\left(p^{0}, p^{A}, q_{A}, q_{0}\right)$.

3 In 5D, this degree of freedom is part of the background Taub-NUT geometry which is frozen.

4 More precisely, the quantum wave function of a hypermultiplet has one supersymmetric ground state corresponding to the unique normalizable self-dual harmonic two form $\omega_{N U T}$. An interesting generalization, on which we hope to report, involves the supersymmetric black ring. 


\section{1. $p^{0}=1$}

In this subsection we take $p^{0}=1$ and then generalize to $p^{0}>1$ in the next subsection. Consider turning on a constant worldvolume $U(1)$ gauge field $F_{w o r l d}=p^{A} \alpha_{A}$ on a IIA $D 6$ brane wrapping the Calabi-Yau $X$. The coupling of $F_{\text {world }}$ to RR potential gives an object in $4 \mathrm{D}$ with charges

$$
\left(1, p^{A}, 3 p^{A} p^{B} D_{A B C},-p^{A} p^{B} p^{C} D_{A B C}\right) .
$$

Solving the attractor equations for such charges, we find simply $C X^{A}=p^{A}, C X^{0}=1$ (see [11] for notation). The leading order macroscopic entropy formula [11] then gives vanishing entropy. This is consistent with the microscopic picture in which there is a unique $F_{\text {world }}$.

Now let us try to understand the 11-dimensional description of this configuration. The M-theory lift is again a Taub-NUT geometry, with nonzero four form flux turned on:

$$
F^{(4)}=\omega_{N U T} \wedge \sum p^{A} \alpha_{A}
$$

$\omega_{N U T}$ here is the unique self-dual harmonic two form on Taub-NUT space [12], and $\alpha_{A}$ is a basis for harmonic Calabi-Yau two-forms. This flux sources D2 charge via the coupling $\int C^{(3)} \wedge F^{(4)} \wedge F^{(4)}$, yielding $q_{A}=3 p^{B} p^{C} D_{A B C}$ as in (5.1). There is a nonzero Poynting vector corresponding to the momentum along the M-theory circle. From the $4 \mathrm{D}$ point of view this is interpreted as D0 charge $q_{0}=-p^{A} p^{B} p^{C} D_{A B C}$ as in (5.1). So, by turning on $F^{(4)}$ as in (5.2), we produce a configuration with $p^{0}=1$, arbitrary $\mathrm{D} 4$ charges, but predetermined D2-D0 charges and no entropy.

To get a configuration with arbitrary D2-D0 charges, we now insert a 5D spinning black hole with charges $q_{A}^{5 D}$ and angular momentum $J_{L}^{3}$ in the middle of this Taub-NUTflux configuration. The exact solution can be found in [2]. This yields a configuration with asymptotic $4 \mathrm{D}$ charges

$$
\left(1, p^{A}, 3 p^{A} p^{B} D_{A B C}+q_{A}^{5 D},-p^{A} p^{B} p^{C} D_{A B C}-p^{A} q_{A}^{5 D}+2 J_{L}^{3}\right)
$$

Notice the extra shift in $D 0$ brane charge coming from placing the charged $5 \mathrm{D}$ black hole in the nontrivial magnetic four form field. This is a higher dimensional generalization of Dirac's observation that a static electric charge in a magnetic field carries angular momentum.

Now we wish to identify the partition function of the $4 \mathrm{D}$ black hole with that of the spinning 5D black hole. 5D black holes doesn't carry $p^{A}$ charge, so in order for this to be 
correct it must be the case that, for the special values of charges given in (5.3), the index $Z_{4 D}$ is independent of $p^{A}$. This can be seen as a consequence of symplectic invariance, as follows.

The index $Z_{4 D}$ is naturally a function of $C X^{\Sigma}=p^{\Sigma}+i \frac{\phi^{\Sigma}}{\pi}$ 《1]. For a cubic prepotential $p^{0}=1$ and $p^{A}=0$, the electric potentials $\phi^{\Sigma}$ are determined from the charges by

$$
\begin{gathered}
q_{0}=-\operatorname{Im} \frac{C D_{A B C} X^{A} X^{B} X^{C}}{\left(X^{0}\right)^{2}}=\operatorname{Re} \frac{D_{A B C} \phi^{A} \phi^{B} \phi^{C}}{\pi\left(\pi+i \phi^{0}\right)^{2}}, \\
q_{A}=3 \operatorname{Im} \frac{C D_{A B C} X^{B} X^{C}}{X^{0}}=-3 \operatorname{Im} \frac{D_{A B C} \phi^{B} \phi^{C}}{\pi\left(\pi+i \phi^{0}\right)} .
\end{gathered}
$$

Now consider the symplectic transformation

$$
C X^{\prime 0}=C X^{0}, \quad C X^{A}=C X^{A}+p^{A} C X^{0},
$$

under which $Z_{4 D}$ is presumed invariant. 0 For the values of the moduli under consideration this results in

$$
X^{\prime 0}=1+i \frac{\phi^{0}}{\pi}, \quad C X^{\prime A}=p^{A}+i\left(\frac{\phi^{A}}{\pi}+p^{A} \frac{\phi^{0}}{\pi}\right) .
$$

Comparing with (5.4) we see that the new charges are related to the old ones by

$$
q_{0}^{\prime}=-\operatorname{Im} \frac{C D_{A B C} X^{\prime A} X^{\prime B} X^{\prime C}}{\left(X^{\prime 0}\right)^{2}}=q_{0}-p^{A} q_{A}-D_{A B C} p^{A} p^{B} p^{C},
$$

and

$$
q_{A}^{\prime}=q_{A}+3 D_{A B C} p^{B} p^{C}
$$

Taking $\left(q_{A}, q_{0}\right)=\left(q_{A}^{5 D}, 2 J_{L}^{3}\right)$, this shift agrees exactly with that encountered in (5.3). Therefore we can use a symplectic transformation to shift from $p^{A}=0$ to arbitrary nonzero $p_{A}$ and $Z_{4 D}$ remains unchanged. Physically this corresponds to the fact that putting a 5D spinning black hole in a background $F^{(4)}$ shifts some charges but does not change the number of microstates.

5 In principle it might transform as a modular form, but this would not affect the leading order computation given here. 


\section{2. $p^{0}>1$}

A similar analysis holds for $p^{0}>1$. The asymptotic charges (5.3) for a spinning black hole become

$$
\left(p^{0}, p^{A}, \frac{3}{p^{0}} p^{A} p^{B} D_{A B C}+q_{A}^{5 D},-\frac{1}{\left(p^{0}\right)^{2}} p^{A} p^{B} p^{C} D_{A B C}-\frac{p^{A} q_{A}^{5 D}}{p^{0}}+2 J_{L}^{3}\right) .
$$

$p^{A}$ can then be shifted away as before via the symplectic transformation

$$
C X^{\prime 0}=C X^{0}, \quad C X^{\prime A}=C X^{A}+\frac{p^{A}}{p^{0}} C X^{0}
$$

\section{Acknowledgements}

This work was supported in part by DOE grant DE-FG02-91ER40654.

\section{Appendix A. Supergravity solutions of spinning black hole in Taub-NUT space}

The Killing spinor equation of $\mathcal{N}=25 \mathrm{D}$ supergravity is

$$
\left[d+\frac{1}{4} \omega_{a b} \Gamma^{a b}+\frac{i}{4 \sqrt{3}} e^{a}\left(\Gamma_{a}^{b c} F_{b c}-4 \Gamma^{b} F_{a b}\right)\right] \epsilon=0
$$

where $e^{a}$ are the frame 1-forms and $\omega_{a b}$ is the spin connection. The metric for the supersymmetric spinning black hole in Taub-NUT space is [2]

$$
d s^{2}=-\left(1+\frac{\tilde{Q}}{R r}\right)^{-2}\left(d t+\frac{\tilde{J} a}{p^{0} R^{2}}\right)^{2}+\left(1+\frac{\tilde{Q}}{R r}\right) d s_{T N}^{2}
$$

where

$$
a=\left(1+\frac{p^{0} R}{r}\right)\left(d x^{11}+p^{0} \cos \theta d \phi\right)-d x^{11}
$$

and $x^{11} \sim x^{11}+4 \pi$. $R$ is the asymptotic radius of the Taub-NUT space and the graviphoton field

$$
F=\frac{\sqrt{3}}{2} d\left[\left(1+\frac{\tilde{Q}}{R r}\right)^{-1}\left(d t+\frac{\tilde{J} a}{p^{0} R^{2}}\right)\right]
$$

Similarly to the calculation of [13], the Killing spinor equations are solved by

$$
i \Gamma^{0} \epsilon=\epsilon
$$

and the self-duality of $d a$ and of the spin connection of Taub-NUT space. 
With a redefinition of variable $r=\rho^{2} / R$, in the limit $R \rightarrow \infty$, the solution (A.2) becomes

$$
d s^{2}=-\left(1+\frac{\tilde{Q}}{\rho^{2}}\right)^{-2}\left[d t+\frac{\tilde{J}}{\rho^{2}}\left(d x^{11}+p^{0} \cos \theta d \phi\right)\right]^{2}+4 p^{0}\left(1+\frac{\tilde{Q}}{\rho^{2}}\right)\left(d \rho^{2}+\rho^{2} d \tilde{\Omega}_{3}^{2}\right)
$$

where

$$
d \tilde{\Omega}_{3}^{2}=\frac{1}{4}\left[d \theta^{2}+\sin ^{2} \theta d \phi^{2}+\frac{1}{\left(p^{0}\right)^{2}}\left(d x^{11}+\cos \theta d \phi\right)^{2}\right]
$$

is the metric on the unit $S^{3} / \mathbf{Z}_{p^{0}}$. (A.6) is nothing but a spinning black hole at the center of the orbifold space $\mathbf{C}^{2} / \mathbf{Z}_{p^{0}}$. Note that the area of the black hole horizon is independent of $R$, and is given by

$$
A=16 \pi^{2} \sqrt{p^{0} \tilde{Q}^{3}-\left(p^{0} \tilde{J}\right)^{2}}
$$

$\tilde{Q}$ and $\tilde{J}$ are related to the standard normalized 5D charges $Q, J$ [⿴囗十 by a rescaling,

$$
Q=2 \pi^{2 / 3} \tilde{Q}, \quad J=2 \sqrt{2} \pi \tilde{J}
$$




\section{References}

[1] H. Ooguri, A. Strominger and C. Vafa, "Black hole attractors and the topological string," arXiv:hep-th/0405146.

[2] J. P. Gauntlett, J. B. Gutowski, C. M. Hull, S. Pakis and H. S. Reall, "All Supersymmetric Solutions of Minimal Supergravity in Five Dimensions," arXiv:hep-th/0209114.

[3] A. Strominger and C. Vafa, "Microscopic Origin of the Bekenstein-Hawking Entropy", Phys. Lett. B 379, 99 (1996) arXiv:hep-th/9601029].

[4] J.C. Breckenridge, R.C. Myers, A.W. Peet and C. Vafa, "D-branes and Spinning Black Holes", hep-th/9602065.

[5] A. Dabholkar, F. Denef, G. W. Moore and B. Pioline, "Exact and asymptotic degeneracies of small black holes," arXiv:hep-th/0502157.

[6] E. Verlinde, "Attractors and the holomorphic anomaly," arXiv:hep-th/0412139.

[7] R. Gopakumar and C. Vafa, "M-theory and Topological Strings - I,II", hepth/9809187; hep-th/9812127.

[8] S. Katz, A. Klemm and C. Vafa, "M-Theory, Topological Strings and Spinning Black Holes", Adv. Theor. Math. Phys. 3, 1445 (1999) arXiv:hep-th/9910181.

[9] R. Kallosh, A. Rajaraman, W. K. Wong, "Supersymmetric Rotating Black Holes and Attractors", Phys. Rev. D 55, 3246 (1997) arXiv:hep-th/9611094.

[10] M. Shmakova, 'Calabi-Yau black holes," Phys. Rev. D 56, 540 (1997) arXiv:hepth/9612076].

[11] A. Strominger, "Macroscopic Entropy of $N=2$ Extremal Black Holes," Phys. Lett. B 383, 39 (1996) arXiv:hep-th/9602111.

[12] R. Gregory, J. A. Harvey and G. W. Moore, Adv. Theor. Math. Phys. 1, 283 (1997) arXiv:hep-th/9708086.

[13] J. P. Gauntlett, R. C. Myers and P. K. Townsend, "Black holes of D $=5$ supergravity," Class. Quant. Grav. 16, 1 (1999) arXiv:hep-th/9810204. 